\title{
Development of a co-operative distributed process mining system for quality assurance
}

\author{
G.T.S. Ho \\ Department of Industrial and Systems Engineering, \\ The Hong Kong Polytechnic University, Hunghom, Hong Kong \\ H.C.W. Lau* \\ Department of Industrial and Systems Engineering, \\ The Hong Kong Polytechnic University, Hunghom, Hong Kong \\ C.K.M. Lee \\ Systems and Engineering Management Division \\ School of Mechanical and Aerospace and Engineering \\ Nanyang Technological University, 50 Nanyang Avenue, Singapore \\ William Ho \\ Operations and Information Management Group \\ Aston Business School, Aston University \\ Birmingham B4 7ET, United Kingdom
}

*Correspondence Address:

Dr Henry Lau

Department of Industrial and Systems Engineering

The Hong Kong Polytechnic University

Hung Hom

Kowloon, Hong Kong

Email: mfhenry@inet.polyu.edu.hk

Tel: (852) 27666628

Fax: (852) 23625267 


\begin{abstract}
In this paper, a co-operative distributed process mining system (CDPMS) is developed to streamline the workflow along the supply chain in order to offer shorter delivery times, more flexibility and higher customer satisfaction with learning ability. The proposed system is equipped with the "distributed process mining” feature which is used to discover the hidden relationships among each working decision in distributed manner. This method incorporates the concept of data mining and knowledge refinement into decision making process for ensuring "doing the right things" within the workflow. An example of implementation is given, based on the case of slider manufacturer.
\end{abstract}

Keywords: Co-operative distributed process mining; quality assurance; online analytical processing 


\section{Introduction}

The recent trend of global manufacturing is to implement system infrastructure that allows analysis on vastly distributed data according to the corporate objectives for making right decisions together with business strategies. Many industries are beginning to explore possible ways to extract useful patterns that may be present in data to facilitate faster, more accurate and better business decisions (Park, Piramuthu \& Shaw, 2001). Process management is primarily concerned with the integration of task and context knowledge in application. Processes vary from place to place and from organization to organization. Future management systems are expected to incorporate process management that enable the operation staff to shift its focus from managing equipment to managing processes. Management will become a distributed, co-operative problem-solving activity (Ray, 2000). Over recent years, Business Process Analysis (BPA), Business Activity Monitoring (BAM), and Business Intelligence (BI) illustrate the desire to offer tools to monitor operational business processes. Process mining can be seen to contribute to this, which aims at extracting information from event logs to capture the business process as it is being executed (van der Aalst \& Weijters, 2004). On the other hand, increasing pressure from the market, technology and society requires manufacturing to be flexible and adaptable. Accordingly, the manufacturing control systems are required to be competent for dealing with various changes and disturbances effectively and efficiently (Huang, et al., 2002).

Although a number of researchers realize the importance of process improvement in manufacturing, only a few solutions exist for building system in real industrial application. It is a challenge to build such a system to cope with different information, 
expertise and decision-making in the distributed manufacturing paradigm for streamlining the workflow. This paper intends to combine Online Analytical Processing (OLAP) with other emergent technologies, such as, neural networks and genetic algorithms, to form a robust and adaptive hybrid system for distributed process mining with improvement ability in a dynamic environment.

\section{Related studies}

In today's competitive environment, markets are becoming more international, dynamic, and customer- driven. Customers are demanding more variety, better quality and service, including both reliability and faster delivery (Lee et al., 2006a; Duclos, Vokurka \& Lummus, 2003). New technologies are required to reduce the operator's cognitive load and achieve more consistent operations (Xia \& Rao, 1999). Manufacturing process management has become more and more complex in the age of mass customization. Still, it is vital to reduce waste and improve quality (Pearson \& Womeldorff, 1999).

To ensure the finished products with high quality and low cost, the finished work piece at each stage should be guaranteed in perfect condition in order to avoid reworking. In reality, the manufacture of a final product takes place on a variety of manufacturing levels in several enterprises. Creating a complete product requires integrating much information from its sub- systems or components. In a virtual enterprise environment, a multi- functional or cross- functional team from different organizations establishes a network of linked decisions with interdependencies (Lee et al., 2006b; Nahm, et.al., 2005). The trend of manufacturing systems is to change from island of automation to enterprise- wise integration, from physical processing workers to information processing workers, and from management of people/ activity to management of information about people/ activities (Zhang, 2001). 
As shown in Figure1, there are many organizations within the enterprise and located world-wide. Poor process decisions from any individual may lead to poor customer satisfaction and the ultimate goal is to achieve better collaboration for making right decision anytime in any enterprise member. To achieve a global optimization in the enterprise network, trade- off analysis should also be conducted in order to generate a set of solutions for benefiting any individual in the enterprise environment. Therefore, an automated management is needed to optimize the whole- valued chain in order to make sure that all the participants have the possibility to act successfully on the market (Fischer, Jahn \&Teich, 2004). There have been many ways of developing IT applications that automate and optimize processes. New computer languages and new methodologies constantly appear, each offering a new centre for the computing universe (Smith, 2003). Expert systems are capable of incorporating human expert's knowledge and analytical ability in a given domain and provide several benefits, including improved decision making, more consistent decision making, reduced design or decision making (Rao \& Miller, 2004). Artificial intelligence (AI) is one of the technologies that have been widely employed to support various business processes in enhancing a company's competitiveness in this global environment (Deng \& Tsacle, 2000). Artificial Neural Network (ANN) is the most commonly used AI techniques for the prediction of process quality (Card, 2000; Kim and Chao, 2000). Unlike the conventional prediction model such as Poisson model, Murphy model, seedy model and negative binomial model, ANN can take fabrication conditions and electrical equipment using parametric data to get an accurate prediction (Kim and Choa, 2000). Although ANN helps to find out the optimal parameter setting for the semiconductor fabrication process and offers engineering "what-if" sensitivity analysis, the globally optimal integrated workflow has not yet achieved. As a result, 
Genetic algorithm is suggested to be integrated in the hybrid system so as to find out the optimal or near optimal workflow by coding a finite-length string. GA takes advantage over the traditional optimization and search techniques that GA can search from a solution space of points instead of a single point. GA therefore can find out the global space rather than confine in a local position. Through the recombination of a selection of potential candidate, GA can get the solution from practical discontinuous function rather than restricting to the continuous function. GA makes use of objective function information and probabilistic transition rules until the optimal solution is approaching.

\section{$<$ Insert Figure 1 $>$}

To many enterprises, Data Mining (DM) can be regarded as a good technological approach to provide sophisticated analysis based on a set of complex data, and has been under active research (Berson \& Smith, 1997; Forcht \& Cochran, 1999). Lian (2002) also pointed out the use of data mining and knowledge discovering can achieve quality improvement in sheet metal assembly industry.

Nowadays, a large scale production information system was implemented to help analyze Quality at Time of Pack in-processing data. This system, known as the Manufacturing Quality System (MQS), is an information system that allows users to access plant data in real time. The graphical representations and statistical analyses provided by the system help operators make production decisions (Stein \& Miscikowski, 1999). However, despite the availability of these methods, the exploratory process analysis for quality prediction and global optimization of supply chain network are not addressed.

The literature review so far indicates that while there exist a number of publications related to the approaches and methodologies to extract useful pattern or information 
for process improvement, the research concerned with the deployment of process quality standard across corporate and distributed database to leverage knowledge in working decisions has not received the attention it deserves. Co-operative distributed process mining is then proposed to create robust, adaptive, and cost- effective approach, and intend to be applied to various industrial applications.

The remainder of this paper is organized as follows. The general infrastructure of proposed Co-operative Distributed Process Mining System (CDPMS) and framework of Process Mining Engine (PME) is introduced in Section 3. In Section 4, the system is developed and considers the application to slider fabrication in order to review the performance of prototype system design and implementation. Experiment results on ANN and GA of slider manufacturing are also stated. Conclusions and future research directions are given in Section 5.

\section{Infrastructure of CDPMS}

The structure of the Co-operative Distributed Process Mining System (CDPMS) architecture is built around different departments within the enterprise as shown in Figure 2. Each department is equipped with the proposed intelligent engine and responsible for optimizing its local workflow by achieving better quality, reducing production cost and management risk. However, today's enterprises operate on a global place and it is a challenge to achieve global optimization within the network. Therefore, the CDPMS is developed for optimizing and encouraging collaborations within and across departmental/ divisional boundaries in a multi-location organization. The essential characteristic of CDPMS is that it views the entire production network as a single entity rather than a series of isolated steps and performs correlation 
analyses and trade-offs among different processes in order to make right decisions for minimizing downtime and higher first pass yield.

$<$ Insert Figure 2>

Basically, the CDPMS consists of two major modules, namely Process Mining Engine (PME) and Dynamic Rule Refinement Engine $\left(\mathrm{DR}^{2} \mathrm{M}\right)$ respectively. Each PME is capable of providing real-time process monitoring, quality prediction and real- time decision support to take corrective actions for continual quality improvements.

Successful operation of CDPMS requires effective communication for collaboration, co-operation, coordination and sharing information. XML is an exciting new technology for exchanging structural information over intranets, extranets and the Internet (Dick, 2000). It is a subset of Standard Generalized Markup Language (SGML), a venerable standard for defining descriptions of structure and content in documents (St. Laurent. \& Cerami, E2001). XML takes the next step providing context and giving meaning to data. It provides a common data exchange format, encapsulating both metadata and data. This allows different applications and databases to exchange information without having to understand anything about each other (Microsoft 1998). The interactions between departments are typed as XML based Process Quality Language for exchanging information independent of content syntax and ontology. The concept of quality improvement adapted in CDPMS is shown in Figure 3 and this is the evolution of Deming's four- step process (PLANDO-CHECK-ACT cycle). This proposed cycle consists of local and global views. The local view mainly focuses on the optimization within the department and the global view focuses on providing a set of solutions for streamlining the whole integrated workflow. 
For the inner circle of the proposed cycle, the first stage (Measurement) is to understand the existing situation and how it is currently performed through different measurements, such as, statistical information, quality information or performance measures with respect to different criteria. Measurement is fundamental to meaningful process improvements. If something cannot be measured, it cannot be improved (Besterfield, 2004). The objective of second stage (Prediction) is to identify the potential quality defects which are going to degrade or fail. The third stage (Correction) is to provide decision supports on how to eliminate poor quality or achieve better resource allocation in order to meet customer expectations. The last step (Action) is to implement the changes on a local scale provided that the developed solutions are approved. After completing all phases mentioned in inner circle, it is required to go to the outer circle for ensuring the enterprise keep productivity with continual quality improvement over time. The improvement in a global view consists of four phases (i) Collection of process log, (ii) Data filtering, (iii) Trade off analysis and (iv) Implementation.

(i) Collection of process log: This phase is to collect the XML based process log from distributed enterprise members and stored in a centralized data warehouse. The content of process log consists of three main parts, such as, process data, performance data and knowledge data.

(ii) Data filtering: The next phase is to convert the process log into information by finding the hidden relationship among used process parameters in different processes and highlighting the undesirable decisions which lead to poor quality.

(iii) Trade off analysis: Before implementing the suggested solutions from PME into global environment, trade-off analysis should be conducted to ensure a set of solutions which are beneficial for the overall manufacturing network based on various 
sources of conflicts. It is because an excellent solution generated in the organization may be poor solution to others due to different built-in goals, point-of-view, budget, time and required specifications.

(iv) Implementation: Implement the suggested solutions and complete the cycle to arrive at 'Doing the right things in each process'. Go back to the first phase to identify the next working task.

\section{$<$ Insert Figure 4>}

\section{1 Framework of Process Mining Engine (PME)}

The framework of PME is composed of three levels which are shown in Figure 4 (Ho et.al., 2006). The first level consists of a measurement module, embraces the practicality of OLAP approach for users to fully understand the current situation at any point of time or other aspects through simple querying. This level should be considered as the preparatory step to the problem solving. The next level consists of a prediction module that is capable of predicting the overview of output quality under given conditions. The bottom-most level is modeled by elementary deductions which can be described by IF-THEN rules which are primarily responsible for giving suggested remedial actions for local improvement. The ultimate goal of this engine is

to eliminate and prevent the problems identified in the local processes. Furthermore, the quality related data from each enterprise member will be exported as XML message for further analysis to arrive at global optimization.

\section{$<$ Insert Figure 4>}

Level 1- Measurement Module: The real time process data can be collected and then transmitted into local database. Whilst the quality data is stored in the database, the further step is to conduct data analysis and data reporting in order to alert or assist process engineers for solving quality problems. The technique of Online Analytical 
Processing (OLAP) is suggested in this module as shown in Figure 4. It facilitates timely access and manipulation of the process data and the application to drill down into data to obtain further information. In the OLAP data cube, it builds up with two elements: fact table and dimension. In the fact table, the measures are defined for data analysis and used the defined measures are used to create user-defined measures (calculated members) which are used on data analysis, e.g. a control chart for keeping a continuing record of a particular quality characteristic in a 'real-time' manner. In the dimension table, different dimension levels are defined to use on different views of data cube which allows engineers to browse the quality information or control charts based on time-to-time variation, piece-to-piece variation and within-piece variation. The other purpose of OLAP in this module is to be the bridge between the measurement and prediction module which carries out data pre-processing (normalization) for artificial neural network.

Level 2- Prediction Module: After observing the real time quality characteristic of work piece in each process obtained in the measurement module, proactive quality prediction is conducted as to ensure the manufactured work piece to be under control. If the predicted quality is out of control, the system will provide some suggestions to refine the relevant process parameters by the improvement module. This prediction module utilizes ANN to learn complex quality data log obtained in each process adaptively without any formulation on the casual relationship between the input and output pattern. The model proposed for quality process prediction is three layered back-propagation networking including an input layer, an output layer and one hidden layer. ANN is composed of many neurons, and each neuron has an adjustable weight factor associated with it. Each neuron is connected to all other neurons in the adjacent 
layer through the weighted connections (Kim, Son \& Yarlagadda 2003). Among neural networks, Multilayer Perceptron (MLP) regression with back- propagation is responsible for predicting quality in the system. Multilayer perceptrons have been applied to solve some difficult and diverse problems by training them in a supervised manner with error back-propagation algorithm. This algorithm is based on the errorcorrection learning rule. The error back propagation algorithm consists of two passes through the different layers of the network which is the forward and backward pass (Haykin, 1999).

Forward pass

$V_{j}(n)=\sum_{i=0}^{m} W_{j i}(n) X_{i}(n)$

where $m$ is the total number of inputs (excluding the bias) applied to neuron $j$, $W_{j i}(n)$ is the synaptic weight connecting neuron $i$ to neuron $j$, and $X_{i}(n)$ is the input signal of neuron $j$.

Backward pass

$W_{j i}(n)=\alpha \Delta W_{j i}(n-1)+\eta \delta_{j}(n) Y_{i}(n)$

where $\alpha$ indicates momentum constant which controls the feedback loop acting around $\Delta W_{j i}(n)$,

$\eta$ indicates learning rate,

$\delta_{\mathrm{j}}(\mathrm{n})$ indicates local gradients that adjust the synaptic weights of the network.

$Y_{i}(n)$ is the output signal of neuron $i$.

In order to facilitate accurate quality prediction within the production line, this module should be learnt by example and analogy. The example is regarded as the historical output quality characteristics with different input process parameters captured. In addition, the value of learning rate, number of layers and number of neurons per layer should be decided to every manufacturing process and is chosen experimentally by the trial and error approach. 
Level 3- Improvement Module: This module carries out decision support in quality optimization for ensuring the output of work piece is accepted with desired quality in each manufacturing sub process. This fuzzy improvement module consists of fuzzy set, fuzzy rule, fuzzy inference and knowledge base as shown in Figure 5. The knowledge base contains the domain knowledge useful for quality problems-solving. The knowledge in this module is represented in a number of rules and stored in an object-oriented structure. Generally, each rule specifies a relation, recommendation, directive, strategy or heuristic and has the IF (condition) THEN (action) structure. When the condition part of a rule is satisfied, the rule is said to fire and the action part is executed (Negnevitsky, 2002). When the crisp value of predicted quality is achieved in the prediction module, it will be fuzzified over all the defined membership function because the predicted value is always a numerical value limited to the universe of discourse. The ranges of the universe of discourses can be determined by experts in the organization. The next step is to apply them to the fuzzy rules stored in the knowledge base, the fuzzy operator (AND or OR) is used to obtain a single number provided that fuzzy rule has multiple antecedents. Combination of the member functions of all rules will be taken and turned into one single fuzzy set. Finally, defuzzification will be carried out to return the crisp number as output. This module enables quality and process engineers to grasp the solutions on how to optimize the related manufacturing processes based on the IF-THEN production rules provided by knowledgeable engineers.

\subsection{XML based Process Quality Language (PQL)}

In order to achieve global optimization of integrated workflow, the CDPMS with connectivity and interactivity is needed. PQL, including Extensible Markup Language 
(XML), acts as the media for data interchange on the information infrastructure regardless of platform and system environment within the organization. XML based Process Quality Language, which is proposed for supporting process information exchange between relational databases within the enterprise network, serves as a process quality standard to highlight the data related to three main components,

The proposed PQL is a set of XML vocabulary which is composed of six main components: Existing workflow, process management responsibility, process requirements, process parameters, process measurements and potential improvement opportunities as depicted in Figure 5. Next, each component of PQL is described followed by the syntax defined via the XML based process quality schema. The major difference between PQL and other existing industrial standards is that PQL includes the linkage of process management and quality performance. As a result, it can specify a process more preciously and analysis techniques can be applied to establish a solid base for well- tuned processes. Other benefits are summarized as follows. First, PQL can be easily exchanged in a geographically dispersed environment and linkages and interactions can be built up between functions/ departments based on the XML data exchange standard. Second, PQL offers the template for recording potentially influential factors on the basis of linked process and quality data and avoids the duplication of process content captured from different departments. The third advantage of PQL is that it can automate the routine aspects of creating process data documents for better control and management of documents.

\subsubsection{Process Quality Schema}

According to (Gryna et al., 2007), effective process and quality improvement can be realized by analyzing process activities, i.e., eliminate non-value-adding work; 
simplify, combine, re-sequence activities; and minimize transfer of material and information especially across departments. To do this includes setting up appropriate process parameters, planning for continuous improvement and periodic assessment. Therefore, the process quality schema is established for defining a strict hierarchy and data types for the process data according to the following methodology that are: (i) Create process quality model and (ii) Transfer the process quality model to XML schema.

(i) To create process quality model:

(a) Identify the existing workflow of the enterprise

- Sequence of processes

(b) Establish process management responsibilities

- Process owner

- Process mission

- Identify customer requirements

(c) Collect the relevant data on process operations

- Operation staff

- Raw materials

- Equipment or machines

- Define the process parameters to be used (e.g. machine setting or working conditions)

- Start time and end time

(d) Define and establish process measures

- Throughput times

- Work In Progress (WIP)

- Process capacity 
- Error rate

(e) Identify potential improvement opportunities

- Identify the root causes of improvement

- Follow through

- Perform periodic process review

(ii) Transfer the process quality model to XML schema

(a) Identify the analogy between XML schema and process quality model:

- Elements

- Behaviour

- Attribute tag within in element tag

- Scriptlet

(b) Construct the XML schema

(c) Consider the following parameters when creating XML schema

- Data fields

- Data types of the attributes

- Maximum length of the elements

- Minimum length of the elements

Identifying the existing workflow of the enterprise is a critical initial stage of process mining. Any industrial enterprise represents a lot of activities, from design of the products, and manufacturing, to marketing and commercial service, management and financing (Bangemann et al., 2006). In order to perform a systematic analysis on how the process really works the sequence of these activities should be identified. Furthermore, the responsibility for the process management is defined by process owners in order to clearly specify the process mission. Having identified the customer needs, each process owner should determine the target outputs in sub processes so as 
to fulfill all requirements. As better process quality is probably driven by correct settings of process parameters, it is necessary to know or record relevant inputs of process, such as machine settings, working conditions or process operators, etc. Some measurements from each process are also needed to reflect how well the process is doing. Different processes may require different measurements to indicate their performance. In deciding which measurements to collect from the process, process effectiveness, process efficiency and adaptability should be considered. In fact,

effectiveness focuses on meeting customer needs (e.g. error rate, accuracy and response time), efficiency addresses meeting the needs of least cost (e.g. output per unit, process yield and work in progress) and adaptability reflects the ability of the process to react positively when process or external conditions change (Gryna et al., 2007). The distributed process data is gathered and analyzed in an integrated manner to improve the yield, eliminate waste and meet customer needs.

\subsection{Framework of $D R^{2} M$}

Each PME carries out decision supports in every department, the distributed process data is then generated and captured for further analysis. $\mathrm{DR}^{2} \mathrm{M}$ is responsible for analyzing the captured process data in order to track and modify the fired rules in each PME which lead to poor quality. This method is a statistical approach for decision rules refinement. It yields improvement trajectory to enhance or modify the fired decision rules. This method involves two main modules, namely online process mining module and rule refinement module. As shown in Figure 6, the PQL message are gathered and imported to the relational database through XML translator. Distributed process mining is carried by adopting OLAP in which data cube is formed by the integration of different databases of PME through the Internet. In the relational 
database, the related or past process data are stored in the predefined cube. The OLAP cube can be used to explore the data by performing slice-and-dice on the data, drilling down and rolling up the database for discovering the level of confidence of fired rules related to inspected quality. In order to highlight undesirable fired rules quickly and efficiently, the dimensions for OLAP cube should be clearly defined, such as, project ID, defect statistic, process parameters and the logs of fired decision rules in each process. To represent the fired rules in the integrated workflow, encoding is the first step to be carried. The first number represents the sequence of processes, and the second alphabet indicates the decision rules fired within the specified process. For example, the code "1AC2B3A4B5CE" means that the combination of Rule A and C is fired in process 1 , Rule B of process 2, Rule A of process 3, Rule B of process 4, and Rule C and E of process 5, etc.

\section{$<$ Insert Figure 6>}

As shown in Figure 7, users can trace the specified combination of fired rules which lead to poor quality. However, in the real situation, not all combinations of fired rules have same quality performance; process engineers must fully understand the fired rules related to the inspected quality and the hidden relationships between processes by drilling the data in different levels. For example: The performance of fired rules can be traced based on time, type of project, customer requirements and decision makers. After highlighting the potential factors, the process engineers can take corrective action and those undesirable rules will be replaced by others in next production. The replacement of rules is carried by reviewing the values of likelihood of sufficiency and likelihood of necessity (Negnevitsky, 2002). In our case, LS is the probability of getting good quality if the specified rule is fired, divided by the probability of getting unsatisfied quality if the specified is fired. LN is the probability 
of getting good quality if the specified rule is not fired, divided by the probability of not getting unsatisfied quality if the specified rule is not fired.

\section{$<$ Insert Figure 7 $>$}

The defined OLAP cube stores all of these statistical data of fired rules and give two possible outcomes- quality is satisfied or quality is unsatisfied. In addition, their likelihood is provided to determine the conditional probabilities of the two hypotheses quality is satisfied or quality is unsatisfied. Higher the values of LS (LS>>1) indicate that the rule strongly supports the hypothesis if the evidence is observed and low values of $\mathrm{LN}(0<\mathrm{LN}<1)$ suggest that the rule also strongly opposes the hypothesis if the evidence is missing (Negnevitsky, 2002). The rules which have the values within specifications, the specified rules will be replaced by others for improvement.

For the trade- off module, a set of desirable rule combinations will be generated for next production based on different customer requirements. The purpose of this is to achieve global optimization of the integrated workflow. Genetic algorithm (GA) is adopted to deal with the search of optimal-solution. The selected sequential numericbased encoding system is appropriate for representing solutions in process of genetic algorithm. The genetic operators include selection operator, crossover operator and mutation operator. Among selection operators like tournament selection, deterministic sampling, remainder stochastic sampling without replacement, stochastic tournament, the elitist function is selected for insuring that the best member of the previous population is carried on to the next generation. According to the findings of Oliver (1987), order crossover (Davis,1985) is deployed for the proposed system for searching local optimal solution. Inconsistent mutation is adopted for maintaining diversity in the population so as to generate a new solution randomly which allows engineers to obtain global optimal solution. Based on the analysis of the weights 
associated with wafer fabrication process suggested by Sugawara (2003), the corresponding weight can be obtained from the weight table.

One of the benefits of using sequential numeric-based encoding system is that in the common genetic operations, the represented code would not generate illegal offspring. The denotation of population is $\mathrm{P}_{0}$, and after $\mathrm{t}$ times of combination the population is denoted as $\mathrm{P}_{\mathrm{t}}$. The iteration from $\mathrm{P}_{\mathrm{t}}$ to $\mathrm{P}_{\mathrm{t}+1}$ is done in three steps. Firstly, evaluation is done on each member from the population $P_{t}$ with the fitness function. Secondly, selecting the members from population $\mathrm{P}_{\mathrm{t}}$ according to their fitness, and makes copy of them to a temporary population P. Thirdly, offspring are generated by recombining the individuals in temporary population $\mathrm{P}$, and the newly generated offspring are placed in the new population $\mathrm{P}_{\mathrm{t}+1}$. The termination criterion is set to reaching the maximum of the defined fitness function. The weighting factor of different criteria (e.g. production cost, production time, reliability and resource utilization, etc) will be assigned by the project decision maker, and its values are passed to the defined fitness function to sum up.

$$
\text { Fitness funtion }=\sum_{i=1}^{I} \sum_{j=1}^{J} W_{j i} R_{j i}
$$

where the resulted fitness function comprises $j=1, \ldots, J$ criterion that are required to be considered with $i=1, \ldots, I$ processes, $W_{j i}$ denotes the weighting assigned to $j^{\text {th }}$ criteria with respect to the $i^{\text {th }}$ process, $R_{j i}$ is the score point of specified fired fuzzy decision rule with respect to the $j$ th criteria in $i^{\text {th }}$ process.

The fitness function with higher value indicates that the particular set of fuzzy decision rules is suitable because it contains the best combination based on the importance of criteria. The newly developed set of combination will be exported into XML format and distributed over the departments in order to arrive at "making no mistake” in any decision. 


\section{A case study: slider manufacturer}

In order to validate the proposed concept, structure and techniques, a rapid conceptual prototype of CDPMS was developed. The prototype was validated in a slider manufacturer and shown to have more expressive power that renders its applicability in more realistic applications involving the quality management.

\subsection{Description of problem}

Magnetic recording areal density has been increasing at about $60 \%$ annual rate over the last 10 years. Higher areal density requires less magnetic spacing between medium and recording head slider (Han, Ryan \& McClure,1999). The manufacturing of slider fabrication involves a number of manufacturing processes which include ion milling, photolithography and vacuum technology, etc. First of all, the wafer should be divided into several blocks by using Diamond Wheel Machines in Wafer-block cutting process. Afterwards, the Lapping machines provide coarse lap the row bars in the End Surface Lapping process. The surface of row bar should be cleaned to prevent any influences in next processes. The SRLG Lapping machine should provide the desired crown, camber, and smooth the surface in the Crown Lapping process. After that, it would be worked in the vacuum process. In the vacuum process, DLC process will be carried for coating the surface of the row bar. DLC is Diamond Like Carbon that uses $\mathrm{Si}+$ and $\mathrm{C}+$ ion sputter on the surface. When the surface is coated, exposure and developing process is needed to expose the slider pattern to be capable of reading/writing information on the hard drive. The row bar would be etched any unprotected films with Ion Milling Machines and cleaned to minimize the potential damage of the next processes. When all procedures in vacuum process are completed, the last step would return to the workshop to separate the sliders with Row Machines. 
Therefore, the output from each process should be in perfect condition in order to meet precise specifications. In this case, there are over hundred manufacturing subprocesses in the whole workflow and are carried in three different production sites as shown in Figure 8. There are three main quality inspection stations which are responsible for documenting the inspected quality data in the database. However, the first pass yield is always undesirable because no analysis is conducted to identify the relationship between quality and operating parameters.

$<$ Insert Figure 8 $>$

CDPMS is then proposed and implemented to monitor each process and ensure all process changes are traced and corrected as soon as possible. A methodology for adopting the principles of $\mathrm{DR}^{2} \mathrm{M}$ to cope with this slider manufacturing process with continual quality improvement is developed. In brief, the local process mining engine is installed in every production site and the overall structure of CDPMS is shown in Figure 9. In CDPMS, the PME is considered as the quantitative tool to deal with the vast amount of captured data from quality control and $\mathrm{DR}^{2} \mathrm{M}$ is the qualitative approach for refining the decision rules in order to streamline the whole integrated workflow.

$<$ Insert Figure 9 $>$

\subsection{Methodology for designing PME}

A methodology with a step-by-step approach is proposed for those who would like to focus on getting the required results without involving too much mathematical analysis. Due to the complexity of slider manufacturing, one of its sub process, DLC coating, is used to illustrate the implementation of PME. The prototype PME 
developed using the Visual Basic 6.0, Microsoft SQL Server 7.0, MATLAB Fuzzy Toolbox and Qnet for Windows.

\subsection{Setting up an OLAP cube for DLC coating process}

Firstly, the process quality data of DLC coating is captured and transferred into the relational data warehouse. The OLAP acts as a multidimensional data model which organizes quality data into a hierarchy that represents levels of details on the data. It allows process engineers to analyze, create and performs quality reports online. This powerful query engine assists engineers to find and retrieve the defected statistics or performance measurement with the dimensions of time, process, work piece and project which can keep monitoring the performance of production line in a real time manner. By using the OLAP approach, the table needed for the query and what data is used to perform calculation is defined in the dimension and measures separately. To perform calculation required by control charts, such as, u charts, c charts, p charts and np charts, calculated member of OLAP is used to meet the specific requirements. Since all the calculation and analyzes pre-compute in OLAP server previously, only a simple multi-dimension expression is needed to construct for retrieving identical result without time delay. Before implementing OLAP approach on real- time quality monitoring and reporting, the data cube must be built in the OLAP server.

\subsubsection{Setting up a NN for DLC coating process}

The neural network of this model serves to provide the recommended change of finished work piece's specification based on what the network has been trained on. As such, it is important that enough data sample for the input and output layers are provided for training purpose. To model this process by neural network, the input and 
output process variables are used and shown in Figure 10. The input process parameters are categorized into 3 types; including (1) customer requirements, (2) previous quality audit data from lapping and (3) DLC process parameters. Generally, there should be some more input parameters to be considered, such as, unexpected performance of manpower and electronic failures of machines. Due to the lack of related data, these factors are assumed to be ignored. In this module, a proprietary neural network simulator called Qnet for Windows was used for the training of the data sets.

\section{$<$ Insert Figure 10>}

As shown in Figure 11, schematic representation of a back-propagating ANN is used. During training process, the network learns the relationship between the data sets mapped to the input and output layers. Based on this capability of machine learning, the trained network is able to induce the change of work piece's specifications in relation to the input parameters which are mapped to the nodes of the input layers. There are 500 sample data sets from DLC coating for training the network. Before feeding the data sets into ANN, the OLAP module carries out data pre-processing which filters out some data which is incomplete and may cause abnormal results. After pre-processing is carried out, the useful data sets are reduced into 409. The Ratio of training data, testing data and production data are 3:1:1 which is the common ratio adopted by most researchers. The design of number of neurons in the input and output layer are 27 and 4 respectively and one hidden layer is chosen to form the optimal model. The value of the learning constant has to be chosen experimentally by the trial-error approach. In this problem, the ideal learning constant and momentum is set to 0.1 and 0.5 and epoch size is 140000 in order to reduce the possibility of any weight oscillation and controls over how much iteration an error adjustment persists. Figure 12 shows a plot of RMS error versus the iterations. The RMS error of training 
set is 0.1055 . The correlation of network outputs and training targets is 0.887 as shown in Figure 13.

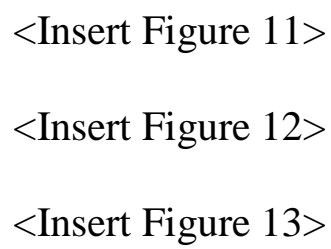

\subsubsection{Setting up a fuzzy decision support level for DLC coating process}

The knowledge base contains the knowledge related to the remedial actions when the quality problems and defects are predicted from trained NN. The fuzzy rules are stored and defined as a conditional statement in IF-THEN form, e.g. IF rate of deposition is short THEN time of sputtering is adjusted to slightly long. This kind of linguistic variables include fuzzy sets, such as, extremely low (EL), slightly low (SL) low (L), medium (M) slightly high (SH) and extremely high (EH) which can greatly reduce a number of production rules. As shown in Figure 14 and 15, the fuzzy rules and fuzzy sets are obtained by interviewing the experts who are familiar with the DLC coating process.

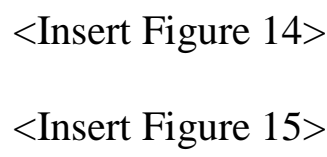

Figure 16 shows the fuzzification and fuzzy inferencing operations and Centre of Gravity (COG) is used for defuzzification in this case. The specified production rules will be replaced by others provided that the values of LS and LN are out of certain level in order to dynamically adapt to changes for continual improvement.

$<$ Insert Figure 16>

\subsection{Result from $\mathrm{DR}^{2} \mathrm{M}$}


In this section, we implement and conduct knowledge discovery among processes. In our implementation, we consider only 11 main processes from three production sites for highlighting the effect of fired decision rules with respect to the yield, production cost and resource utilization of finished products. The $\mathrm{DR}^{2} \mathrm{M}$ was run after collecting the PQL message from three different production sites. After performing the spatial analysis (as shown in Table 1,2 and 3), it shows that specified decision rules combination may affect addressed factors, and these results can help modify the values of LS and LN in order to make accurate decisions for next production. Furthermore, it indicates that the specified machine and operators under specified time period will lead to higher probability of rework. This gives a signal to process engineers to adjust the parameter settings or setup for improvement based on different requirements of customers. It can help yield a fundamental type of management needed for controlling operations. This approach creates focus, triggers corrective action for improving strategic choices. After having this mining approach, the reasons which lead to poor customer satisfaction can be traced by the robust relational OLAP cube.

$$
\begin{aligned}
& <\text { Insert Table 1> } \\
& \text { < Insert Table 2> } \\
& <\text { Insert Table 3> }
\end{aligned}
$$

Beyond the above process, there is also enabling integrated processes meant to improve or empower ordinary workflow by searching the optimal combination of decision rules within the whole workflow. In reality, there are trade-off relations among these. In some instances, optimizing the local process reduces benefit of global quality. Knowing exactly what these trade-off relations allows management to optimize around the enterprise of output quality. 
To analyze the feasibility of the proposed GA approach, a number of eleven subprocesses of slider manufacturing are chosen by enumerating all combinations of fuzzy decision rules, where the average number of rules in each process knowledge base is ten. In reality, there are over hundreds of sub- processes within the integrated workflow, GA approach is then a good way to search the ideal combination of decision rules based on the company's criteria for streamlining process quality. The fitness function in Eq. (3) is presented as an evaluation function. The performance of fuzzy decision rule combinations with their associated criteria score point is then fed back to the GA to control how the solution space is searched to promote the global workflow quality. Three important criteria in this case study (production cost, yield and resource utilization) are considered in evaluating derived combinations. The score point is ranged from 0 to 1 , where higher values, better performance achieved. The details of GA operations are illustrated as below.

\section{Chromosome representation}

Figure 20 depicts the chromosome encoding method used. Assume that $N$ processes are considered. There are a number of rules involved but not all rules are required in each process. Then, the decision whether a rule is needed or not is encoded into a gene with value 1 or 0 respectively for each process. For examples as shown in the Figure 20, since the gene values corresponding to the rules A and B are 1 but those to the rules $\mathrm{C}$ and $\mathrm{D}$ are 0 in the process 1 , only the former two rules are chosen. Based on the gene representation in rules $\mathrm{A}$ and $\mathrm{B}$, Figure 17 shows a practical example to illustrate that the consequence for the process 1 is related to pressure of deposition, pressure of pre-sputtering, pressure of silicon sputtering and fixture angle of row bar 
rather than beam current, beam voltage and extrusion coefficient. The same inference method is also applies to other processes.

$<$ Insert Figure 17>

\section{Selection}

Selection is the process which introduces the selection pressure into the population so as to favour fitter individuals. The selection method used is a roulette wheel selection which is shown in Figure 18.

$<$ Insert Figure 18>

The steps of its implementation are given as follows.

Step 1: Calculate the selection probability $\left(P_{s}\right)_{i}$ for an individual $i$ by

$\left(P_{s}\right)_{i}=\frac{g_{i}}{\sum_{k \in C_{h}} g_{k}}, \forall i \in C_{h}$

where $g_{i}$ is the fitness value of the individual $i$ obtained by the OPI fitness function.

In fact, $\left(P_{s}\right)_{i}$ determines the arc length and hence area of the pie for the individual $i$. The higher the value of $\left(P_{s}\right)_{i}$, the longer the arc length and therefore the larger the area of the pie for the individual $i$. As a result, the individual $i$ has a higher chance of being selected.

In Figure 18, chromosomes 2, 3 and 5 have larger portions of the wheel while chromosomes 1, 4 and 6 have smaller portions. Therefore, the former chromosomes have a larger chance of being selected, while the latter 
chromosomes have a lower chance. Note that it is possible that if a chromosome is selected once, it can still be chosen again.

Step 2: Calculate the cumulative probability $\left(P_{\text {cum }}\right)_{i}$ for an individual $i$ by

$$
\left(P_{\text {cum }}\right)_{i}=\sum_{k=1}^{i}\left(P_{s}\right)_{k}, \forall i \in C_{h}
$$

Step 3: Generate $R_{m \times 1}$

Step 4: The pseudo code of the selection process is given as follows.

begin

$$
\left(P_{\text {cum }}\right)_{0}=0
$$

$k=1$; $\quad$ // Initialize the chromosome

counter $k$ while $(k \leq M)$

$l=1 ; \quad$ // Initialize the chromosome counter $l$

while $(l \leq M)$

$$
\text { if }\left(\left(P_{\text {cum }}\right)_{l-1}<r_{k} \leq\left(P_{\text {cum }}\right)_{l}\right)
$$

mating pool;

pick chromosome $l$ from the parent pool to the break;

end if;

$l=l+1$

// Consider the next chromosome $l$

end while;

chromosome $k$

$k=k+1$

// Consider the next

end while;

end

The purpose of this selection process is to select $M$ individuals from the parent pool for transfer to the mating pool. With reference to Figure 18, if a random number is smaller than $\left(P_{\text {cum }}\right)_{1}$, the chromosome 1 in the parent pool will be picked. In case it lies between $\left(P_{\text {cum }}\right)_{1}$ and $\left(P_{\text {cum }}\right)_{2}$, then chromosome 2 will be 
selected and so on. After filling the mating pool with $M$ chosen individuals, the selection process is completed.

\section{Crossover}

At first, we require $N_{p}$ random numbers to be generated between 0 and 1 to decide which pair(s) of chromosomes will be chosen for performing the crossover where $N_{p}$ is the population size. If a random number corresponding to a chromosome is less than the crossover probability, $p_{c}$, that chromosome will be chosen to perform crossover with the others. In the simulation, different types of crossover, one-point crossover, two-point crossover and uniform crossover, are tested. Because the better results are obtained by using uniform crossover, it is the process of crossover used and we will introduce its operation only.

Figure 19 demonstrates the operation of uniform crossover. In the figure, we generate a mask containing the elements of a binary sequence restricted to 0 or 1 only. Each binary number corresponds to one gene of the chromosomes, parents 1 and 2 . If a binary number corresponding gene is 1 , the genes of parents 1 and 2 are exchanged. Otherwise, they remain unchanged.

$$
<\text { Insert Figure 19> }
$$

\section{Mutation}

To perform a mutation, we produce $\left(N_{p} \times Q\right)$ random numbers, where $Q$ is the maximum chromosome length. When a random number corresponding to a gene in the chromosome is less than the mutation probability, $p_{m}$, the gene will be mutated. Figure 20 illustrates the operation of mutation. The highlighted genes are chosen and performed the bit-flip mutation (i.e. 0 is changed to 1 and vice versa). 
There were a number of works studying the selection of GA parameters (i.e. population size, crossover rate and mutation rate). Hesser et al. (1989) and Grefenstette (1986) tackled the determination of the range of the population size for obtaining the optimal performance of GA. They showed that population size between 30 and 110 can successfully drive GA to acquire such performance. Similarly, De Jong (1975) and Schaffer (1989) dealt with testing the range of crossover rate and mutation rate. Better results can be got by using crossover rate between 0.6 and 0.9 as well as mutation rate between 0.001 and 0.01 .

\section{Fitness Functions}

The fitness function is used to evaluate each member from the population $\mathrm{P}_{\mathrm{t}}$. In the evaluation function, $W_{j i}$ denotes the weighting assigned to $j^{\text {th }}$ criteria with respect to the $i^{\text {th }}$ process, $R_{j i}$ is the score point of specified fuzzy decision rule with respect to the $j^{\text {th }}$ criteria in $i^{\text {th }}$ process.

Suppose the $1^{\text {st }}$ criterion in process 1 is cost minimization and the weight of the $1^{\text {st }}$ criteria is 0.8 . Since the consequence of Fuzzy rule A shown in Figure 14 is that pressure of deposition is adjusted to low and pressure of pre -sputtering is adjusted to medium and pressure of silicon sputtering is adjusted to medium. Since pressure is adjusted to small, energy used is relatively low so as to achieve cost minimization. The $2^{\text {nd }}$ criterion in process 1 is minimization of set up time and the weight of the $2^{\text {nd }}$ criteria is 0.2 . Since the Fuzzy rule B is that fixture angle of row bar is adjusted to low while Fuzzy rule A is fixture angle of row bar is adjusted to very low which requires comparatively longer setup time. After calculation the sum of weight of criteria for process 1 , Rule A is fitter than Rule B. 


\subsection{Application of CDPMS for trade-off requirements of different departments}

Co-operative distributed process mining system (CDPMS) is developed to streamline the workflow along the production process in order to offer shorter delivery times, more flexibility and higher customer satisfaction with machine-learning. It is common to see that department of sales and marking and division of manufacturing have different point of view to fulfill customer satisfaction. Sales and marketing department would like to deliver the high-quality customized goods in small batch for the customer and react to customer needs responsively in accordance with the changing customers' needs. However, it may lead to the change of production plan and layout to enhance the production efficiency. However, the manufacturing department prefers to keep the layout of shop floor unchanged and takes advantage of the scope of economic by producing products in a large batch. Instead of keeping the unit cost low by increasing the batch size, manufacturers can adopt the proposed system to predict the output quality under given conditions so as to minimize the scrap rate, which in turn, minimizes the cost.

\subsection{Evaluation of PME and $D R^{2} M$}

Instead of measuring how good PME and $D R^{2} M$ are, authors measure how PME and $D R^{2} M$ can achieve quality assurance. There are two radar charts which illustrate the important categories of performance and make visible concentration of strengths and weakness compared with the traditional approach of slider manufacturing implementation. Each spoke is subdivided into a number of increments established in the rating scale. The scoring range is defined as 0 to 10 with 10 being full performance. The operators and process engineers were invited to participate in the prototype evaluation. The response rate was about one- fifth of the population. 82 
responses are included in the summary statistics for the evaluation of PME and $\mathrm{DR}^{2} \mathrm{M}$ respectively.

For the radar chart (Figure 21) shown in left hand side, those subjective attributes are reserved to the measurable dimensions which are (1) First pass yield (2) Level of control (3) Schedule are met (4) Level of visibility (5) Quality (6) Operator satisfaction (7) Efficiency process tracking. Seven dimensions are represented by seven axis of the radar chart in Fig. 24. Referring to the radar chart, it is found that the first pass yield is enhanced. Operators found that PME helps taking proactive actions to eliminate the quality problems in various areas. Therefore, the schedules and quality are always met. Furthermore, the customers' unsatisfied orders can be traced because the process log are captured in each process and stored in a centralized database.

$<$ Insert Figure 21 $>$

For the radar chart shown in right hand side, $\mathrm{DR}^{2} \mathrm{M}$ shows significant improvement in customer satisfaction compared with the traditional approach. It can shorten the manpower usage by automating the information sharing within the enterprise. In addition, the product development cycle is greatly reduced because the previous knowledge is captured and reused by the system. However, the cost of the implementation of $\mathrm{DR}^{2} \mathrm{M}$ is yet to be considered because the setup cost of server and database is large.

In summary, certain extent of improvement has been shown in the slider manufacturing operation and the overall impact due to be adoption of the proposed system can be realized. Further research is needed to substantiate the proposed approach subject to future funding support. 


\section{Conclusions}

This paper presents a new way for quality assurance using a new process mining approach- Cooperative Distributed Process Mining. The major contribution of the proposed approach is to enhance customer satisfaction by discovering the relationship between working decisions and quality by quantifying the knowledge extracted from operations into fuzzy rules. In the proposed system, GA is included to find out best fit rules for achieving better finished quality so as to realize the hidden pattern and relationship between operations in different disciplines that can achieve the goal of quality assurance. It is recommended for the researchers to utilize the innovative information technologies to create values for customers with TQM principles that can help the organization to provide ultimate customer satisfaction. Further research on the infrastructural framework, particularly relating to the synergetic combination, the technologies, is needed in order to leverage the intelligence level of the process mining engine.

\section{Acknowledgements}

The authors wish to thank the Research Office of the Hong Kong Polytechnic University for the support of this research project. 


\section{References}

Berson, A., \& Smith, S. J. (1997). Data warehousing, data mining, and OLAP, New York: McGraw-Hill.

Besterfield, Dale H. (2004). Quality control seventh edition: Pearson Pretice Hall

Davis L. (1985) Applying adaptive algorithms to epistatic domains. Proceedings of the international joint conference on artificial intelligence. pp.162-164

Dick, Kevin (2000). XML A Manager’s Guide: Addison- Wesley.

Deng, P.-S. \& Tsacle, E.G. (2000). Coupling genetic algorithms and rule-based systems for complex decisions. Expert Systems with Applications (2000) 209-218

Ducios, Leslie K., Vokurka, Robert J. \& Lummus, Rhonda R. (2003). “A conceptual model of supply chain flexibility”, Journal of Industrial Management \& Data Systems Vol. 103 Number 62003 pp.446-456.

Fischer, Qijun., Jahn, Hendrik. \& Teich, Tobias. (2004). Optimizing the selection of partners in production networks. Robotics and Computer- Integrated Manufacturing 20 (2004) 593-601.

Forcht, K.A. \& Cochran, K. (1999). "Using data mining and data warehousing techniques”, Journal of Industrial Management \& Data Systems Vol. 99 Number 5 1999 pp.189-96.

Gryna, Frank M., Chua, Richard, C.H. \& Defeo, Joseph A. (2007) Juran's quality planning and analysis: For enterprise quality, fifth edition: McGraw- Hill, New York.

Han, H., Ryan, F. \& McClure, M. (1999). "Ultra-thin tetrahedral amorphous carbon film as slider overcoat for high areal density magnetic recording. Surface and Coating Technology”, Vol 120-121, pp.579-584.

Haykin, Simon (1999). Neural Networks: A Comprehensive Foundation, Second Edition: Prentice- Hall.

Ho, G.T.S.; Lau, H.C.W.; Lee, C.K.M.; Ip, A.W.H. and Pun, K.F. An intelligent production workflow mining system for continual quality improvement, The International Journal of Advanced Manufacturing Technology, Vol. 28 No 7-8, p792809 (2006)

Huang, Biqing., Gou, Hongmei., Liu, Wenhuang., Li, Yu. \& Xie, Min. (2002). A framework for virtual enterprise control with the holonic manufacturing paradigm. Computers in industry 49, 299-310.

Kim, Ill- Soo, Son, Joon- Sik \& Yarlagadda Prasad K.D.V (2003). “A study on the quality improvement of robotic GMA welding process. Robotics and Computer Integrated Manufacturing”, Vol. 19 pp. 567-572. 
LEE, C. K.M., G.T.S. Ho, LAU, H.C.W. and YU , K.M. (2006a) “A dynamic product information schema for supporting responsive product lifecycle management", Journal of Expert Systems with Application, Vol. 31, No. 1, p. 30-40

Lee, C.K.M.; LAU, H.C.W.; B.P.K. Leung G. T. S. Ho and K. L. Choy (2006b) "Enhancing product development through a format-independent information exchange platform”, International Journal of Production Research, Vol.44, No. 23, pp 5197-5222

Lian, J., Lai, X.M., Lin, Z.Q. \& Yao, F.S . (2002). Application of data mining and process knowledge discovery in sheet metal assembly dimensional variation diagnosis. Journal of Materials Processing Technology 129 (2002) 315- 320.

Microsoft Corporation (1998). XML: Enabling Next Generation Web Applications.

Nahm, Y.-E. \& Ishikawa, H. (2005). A hybrid multi-agent system architecture for enterprise integration using computer networks. Robotics and Computer- Integrated Manufacturing 21 (2005) 217-234.

Negnevitsky Michael. (2002). Artificial Intelligence: A guide to intelligent systems, first edition: Addison Wesley.

Oliver IM. Smith DJ, Holland JRC. (1987). A studying of permutation crossover operators on the traveling salesman problem. Proceedings of the second international conference on genetic algorithms. Hillsdale, NJ: Lawrence Erlbaum Associate; pp.224-230

Park, Sang C., Piramuthu, Selwyn. \& Shaw, Michael J. (2001). Dynamic rule refinement in knowledge-based data mining systems. Decision Support Systems 31 (2001) 205-222.

Pearson, Tom., Womeldorff, Tom.(1999) Predictive Process Management. Manufacturing Systems, Novemeber 1999.

Ray, Pradeep. (2000). Cooperative management of enterprise networks: Kluwer Academic/ Plenum Publishers, New York.

Rao, Mohan P., Miller, David M. (2004). "Expert systems applications for productivity analysis”, Journal of Industrial Management \& Data Systems Vol. 104 Number 92004 pp.776- 785.

Smith, Howard. (2003). Business process management- the third wave: business process modeling language (bpml) and its pi-calculus foundations. Information and Software Technology 45 (2003) 1065-1069.

St. Laurent, S. \& Cerami, E. (2001). Building XML Applications: McGraw- Hill.

Stein, E.W. \& Miscikowski, D.K . (1999). FAILSAFE: supporting product quality with knowledge- based systems. Expert Systems with Applications 16 365-377. 
Sugawara E.; Fukushi M. \& Horiguchi S.(2003) Fault Tolerant Multi-layer Neural Networks with GA Training, Defect and Fault Tolerance in VLSI Systems, 2003. Proceedings. 18th IEEE International Symposium on 3-5 Nov. 2003, 328 - 335

van der Aalst, W.M.P \& Weijters, A.J.M.M. (2004). Process mining: a research agenda. Computers in industry 53 (2004) 231- 244.

Xia, Qijun. \& Rao, Ming. (1999). Dynamic case-based reasoning for process operation support systems. Engineering Applications of Artificial Intelligence (1999) 343-361.

Zhang, Qingyu. (2001). “Object- oriented database systems in manufacturing: selection and applications”, Journal of Industrial Management \& Data Systems Vol. 101 Number 32001 pp.446-456.

J. Hesser, R. Manner, O. Stucky (1989). “Optimization of steiner Trees using genetic algorithms”, Proceedings of the Third International Conference on Genetic Algorithms, Arlington, pp. 231 - 236, June 1989.

J. J. Grefenstette (1986). “Optimization of control parameters for genetic algorithms”, IEEE Transactions on Systems, Man and Cybernetics, vol. 16, no. 1, pp. 122 - 128, 1986.

K. A. De Jong (1975). “An analysis of the behavior of a class of genetic adaptive systems,” Ph.D. dissertation, University of Michigan, Ann Arbor, 1975.

J. D. Schaffer, R. A. Caruana, L. J. Eshelman, and R. Das (1989). “A study of control parameters affecting online performance of genetic algorithms for function optimization," in Proceedings of the third International Conference on Genetic Algorithms. San Mateo, CA: Morgan Kaufmann, 1989, pp. 51 - 60. 


\section{University Library}

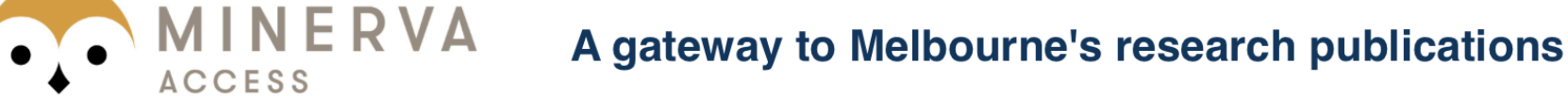

Minerva Access is the Institutional Repository of The University of Melbourne

Author/s:

Ho, GTS;Lau, HCW;Kwok, SK;Lee, CKM;Ho, W

Title:

Development of a co-operative distributed process mining system for quality assurance

Date:

2009-01-01

Citation:

Ho, G. T. S., Lau, H. C. W., Kwok, S. K., Lee, C. K. M. \& Ho, W. (2009). Development of a co-operative distributed process mining system for quality assurance.

INTERNATIONAL JOURNAL OF PRODUCTION RESEARCH, 47 (4), pp.883-918. https:// doi.org/10.1080/00207540701441939.

Persistent Link:

http://hdl.handle.net/11343/118672 